\title{
Relación entre periodontitis y disfunción eréctil: đrealidad o mito?
}

\section{Relationship between periodontitis and erectile dysfunction: reality or myth?}

\author{
Sayra Krystell Aguilar-Manrique,* Celia Elena del Perpetuo Socorro Mendiburu-Zavala, ${ }^{\ddagger}$ Josué Carrillo-Mendiburu $^{\S}$
}

\section{RESUMEN}

Introducción: Se realiza una revisión bibliográfica con base en artículos científicos con la finalidad de exponer la posible relación entre periodontitis (PD) y disfunción eréctil (DE), así como las variables contundentes que se encuentran implicadas. Material y métodos: Como estrategia de búsqueda primaria se emplea una combinación de vocabulario controlado (erectile dysfunction and periodontal disease) y términos de texto libre basado en la estrategia de búsqueda en MEDLINE a través de PubMed. Como estrategia de búsqueda secundaria se revisaron las referencias bibliográficas contenidas en los artículos seleccionados. Resultados: Diez estudios de casos y controles encontraron asociaciones positivas significativas entre estas dos condiciones. Conclusión: La evidencia de la literatura indica un vínculo positivo entre la $\mathrm{PD}$ y la $\mathrm{DE}$.

Palabras clave: Periodontitis, disfunción eréctil, disfunción endotelial.

\section{ABSTRACT}

Introduction: A bibliographic review was carried out in scientific articles in order to expose the possible relationship between periodontitis (PD) and erectile dysfunction (ED), as well as the overwhelming variables that are involved. Material and methods: A combination of controlled vocabulary (erectile dysfunction and periodontal disease) and free text terms based on the MEDLINE search strategy through PubMed was used as the primary search strategy. As a secondary search strategy, the bibliographic references contained in the selected articles were reviewed. Results: Ten case-control studies found significant positive associations between these two conditions. Conclusion: The evidence in the literature indicates a positive link between $P D$ and $E D$.

Keywords: Periodontitis, erectile dysfunction, endothelial dysfunction.

\section{INTRODUCCIÓN}

$\mathrm{L}$ a periodontitis (PD) y la disfunción eréctil (DE) comparten factores de riesgo y afecciones sistémicas asociadas, como el tabaquismo, la diabetes mellitus y la enfermedad de las arterias coronarias. La DE y la PD pueden ser síntomas de estas afecciones y pueden actuar como marcadores de progresión en enfermedades sistémicas. ${ }^{1}$ En los últimos años se han realizado constantes estudios que exponen la posible relación entre ambas.

La PD es una enfermedad inflamatoria disbiótica con un impacto adverso sobre la salud sistémica. Estudios re- cientes han proporcionado información sobre la aparición y persistencia de comunidades microbianas orales que pueden mediar la patología inflamatoria en sitios locales y distantes, como es la disfunción endotelial sistémica, que conduce a la enfermedad vascular. Es sabido que las afecciones vasculares son las causas más comunes de disfunción eréctil. ${ }^{2,3}$

\section{Periodontitis}

La PD es una enfermedad que compromete la integridad de los tejidos que sostienen los dientes, que incluyen

\footnotetext{
* Práctica privada. Egresada de la Facultad de Odontología de la Universidad Autónoma de Yucatán.

‡ Facultad de Odontología de la Universidad Autónoma de Yucatán. Cuerpo Académico de Odontología Restaurador. Docente.

$\S$ Práctica privada. Especialista en Otorrinolaringología y Cirugía de Cabeza y Cuello. Egresado de la Universidad Nacional Autónoma de México.

Recibido: 25 de Agosto de 2020. Aceptado para publicación: 17 de Noviembre de 2020.
}

Citar como: Aguilar-Manrique SK, Mendiburu-Zavala CEPS, Carrillo-Mendiburu J. Relación entre periodontitis y disfunción eréctil: ¿̇realidad o mito? Rev ADM. 2020; 77 (6): 306-311. https://dx.doi.org/10.35366/97619

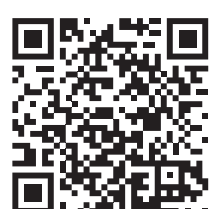


la encía, el ligamento periodontal y el hueso alveolar, y se conocen colectivamente como el periodonto. Esta patología se hizo frecuente después de la domesticación de plantas y animales en las sociedades neolíticas (hace aproximadamente 10,000 años) cuando el microbiota oral experimentó un cambio de composición distinto, con un aumento en la frecuencia de Porphyromonas gingivalis y otras especies asociadas con periodontitis, en comparación con sociedades de cazadores-recolectores anteriores. $^{2}$

En la enfermedad prevalece un biofilm en el que los microorganismos anaerobios Gram negativos dominan. La gravedad de la rotura periodontal está vinculada con la respuesta inflamatoria del huésped. Los episodios diarios de bacteremia o la diseminación de endotoxinas originarias desde el foco periodontal pueden inducir la activación sistémica de la respuesta inflamatoria debido a que hay una producción de citocinas proinflamatorias. Estas citosinas activan aún más las respuestas inflamatorias (RI), lo que resulta en una baja calidad sistémica de la regulación de las RI crónicas, que implican a la interleucina (IL) 6, la proteína $C$ reactiva, prostaglandina E2 (PGE2) y el factor de necrosis tumoral alfa (TNF $\alpha$ ), así como también incluye la activación de las células inflamatorias y las endoteliales, que pueden resultar en la disfunción endotelial. ${ }^{4.5}$

\section{Disfunción endotelial}

En la actualidad la disfunción endotelial se considera una de las primeras manifestaciones de la enfermedad vascular y la ateroesclerosis. El endotelio regula la interacción de las células y las proteínas circulantes con las células residentes en la pared vascular, ejerciendo un papel central como sensor y transmisor de señales. Éste protege la pared arterial frente al desarrollo de lesiones y contribuye a la homeostasis vascular a través de ese control continuo de los estímulos que recibe y la adaptación de su estado funcional. Diversos factores pueden modificar las funciones del endotelio y provocar lo que se conoce como disfunción endotelial, que es el desequilibrio en la biodisponibilidad de sustancias activas de origen endotelial que predispone a la inflamación, la vasoconstricción y el incremento de la permeabilidad vascular, o que puede facilitar el desarrollo de ateroesclerosis, agregación plaquetaria y trombosis. ${ }^{6}$

La disfunción endotelial resultante de la inflamación crónica se asocia con un aumento en las especies reactivas de oxígeno (ROS). Este exceso de producción de ROS resulta en una mayor inactivación en el óxido nítrico. ${ }^{7}$

\section{Fisiología de la erección}

El pene es el órgano masculino que permite la micción y las relaciones sexuales. Su anatomía posee partes distintivas de un esqueleto hidrostático, entendiéndose como esqueleto a cualquier sistema que transmite fuerzas. La parte hidrostática funciona con dos elementos: el fluido y una pared de tejido circundante reforzada con proteínas fibrosas, constituyendo una interacción crucial para brindar soporte. Las dos estructuras hidrostáticas eréctiles son el cuerpo cavernoso y esponjoso. El cuerpo cavernoso se encuentra en la parte dorsal del pene y es un sistema de alta presión responsable del incremento del tamaño del pene y la rigidez a la flexión durante la erección. Está compuesto de un espacio vascular revestido por células endoteliales, rodeado por una pared gruesa de tejido colágeno llamado túnica albugínea. El cuerpo esponjoso, más pequeño, contiene la uretra y se expande distalmente para formar el glande. A pesar de que también se somete a la vasodilatación durante la erección, no es responsable de la rigidez del pene. Cuando el pene es visto en sección transversal, se observa una parte central de tejido eréctil y esponjoso que se llena de fluido (sangre), y está rodeado por una pared de tejido rica en una proteína estructural denominada colágeno. ${ }^{8}$

La erección es el resultado de la interacción de factores hormonales, vasculares, neurológicos, sinusoidales, musculares y psicológicos que culmina en un aumento del flujo arterial dentro del lecho del pene hipogástrico, seguido de la activación del mecanismo venooclusivo de los cuerpos cavernosos. Su principal controlador es el sistema nervioso autónomo, siendo esto un fenómeno eminentemente neurovascular con un mediador universal que es el óxido nítrico. ${ }^{8,9}$

Durante la flaccidez, el músculo liso cavernoso (por efecto del sistema adrenérgico sobre receptores alfa1 postsinápticos y alfa2 pre- y postsinápticos) está contraído, lo que hace que el flujo de sangre sea bajo. Cuando se produce el estímulo erótico y la excitación sexual, por vías parasimpáticas y oxitocinérgicas, se envía información a las neuronas no adrenérgicas/no colinérgicas y al endotelio de las arterias del pene, se libera óxido nítrico a partir del aminoácido L-arginina-vía citrulina- por efecto de la enzima óxido nítrico sintetasa, provocando que los vasos del pene se dilaten por acción del guanilato ciclasa, lo que resulta en un nivel aumentado de GMPc, con el ingreso de una mayor cantidad de flujo sanguíneo. A su vez, se produce por un doble mecanismo, uno activo y otro pasivo, la compresión de las venas por donde retorna la sangre, generando, como consecuencia, una rigidez suficiente. ${ }^{6}$ 
Durante el proceso de la erección se reconocen seis fases hemodinámicas diferentes: flaccidez, erección latente (preerección), tumescencia, erección máxima, rigidez y detumescencia. ${ }^{10}$

\section{Disfunción eréctil}

Las dificultades en la respuesta sexual han sido siempre motivo de preocupación y sufrimiento para las personas que las padecen y sus parejas. Una de las alteraciones de la respuesta sexual en el hombre que más atención demanda es la $\mathrm{DE}$, debido a que compromete de manera significativa el desempeño sexual, la satisfacción personal, el compromiso con la pareja y la autoestima de quienes lo sufren. ${ }^{11}$

La DE se define como la incapacidad persistente o recurrente para completar o continuar una erección suficiente para un desempeño sexual satisfactorio. Es una condición común y no es un trastorno que ponga en peligro la vida, pero puede influir en la rutina diaria, las actividades sociales, el bienestar y la calidad de vida del paciente. En la actualidad, se considera ampliamente un síntoma de enfermedad subyacente en lugar de una enfermedad en sí misma. ${ }^{12}$ El mayor número de casos de disfunción eréctil tienen origen orgánico, principalmente por enfermedades vasculares, pero también está asociada a alteraciones estructurales, factores psicológicos, neurológicos y hormonales. ${ }^{11} \mathrm{La}$ DE está relacionada con el deterioro de la función endotelial en la enfermedad cardiovascular, y se cree que este mismo mecanismo está involucrado en su desarrollo. ${ }^{13}$ De igual forma, se ha asociado con comorbilidades médicas y psicosociales. Para el diagnóstico, es importante realizar una entrevista exhaustiva en la que se aplique el método clínico con el fin de identificar todas las variables biológicas, psicológicas y sociales que puedan constituir la causa de este padecimiento. ${ }^{11}$

En el mundo hay alrededor de 152 millones de varones con DE, y se estima que esta cifra aumentará a 322 millones para el año 2025. ${ }^{14}$ En México, dentro de la consulta privada urológica, sólo 15\% acepta tener problemas de este tipo, cuando en realidad está documentado que hasta $52 \%$ de los hombres mayores de 40 años lo padecen. ${ }^{15}$ De estos últimos, afecta en $55 \%$ en forma leve, a $35 \%$ de manera moderada y a $10 \%$ en forma severa. ${ }^{14}$

El bloqueo de las arterias cavernosas por la ateroesclerosis, así como el deterioro de la función endotelial o la relajación del músculo liso, pueden provocar la disfunción eréctil. La disfunción endotelial es el evento clave en la fisiopatología de la disfunción eréctil, y también se encontró en otros lechos vasculares en hombres afectados con disfunción vascular del pene. La ateroesclerosis comienza primero en los vasos pequeños, como la vasculatura del pene, y luego en las arterias más grandes, como las coronarias. ${ }^{7}$

\section{Relación entre PD y DE}

La inflamación en la disfunción endotelial causada por la PD es la base principal de la asociación entre ésta y la DE. ${ }^{16}$ Sin embargo, se han postulado muchas hipótesis. La pérdida de órganos dentarios causada por la PD podría afectar la capacidad de masticación, lo que Ilevaría a una dieta alterada, lo que podría aumentar el riesgo de disfunción endotelial. De manera alternativa, la disfunción endotelial podría ser el resultado del aumento de la respuesta inflamatoria asociada con la PD. ${ }^{13}$

Se han propuesto tres mecanismos para explicar esta relación. Primer mecanismo: la inflamación crónica en la disfunción endotelial se asocia con un aumento de especies reactivas de oxígeno. El exceso conduce a un aumento en el óxido nítrico. La inactivación y el daño al sistema antioxidante pueden contribuir a la disfunción endotelial en pacientes con periodontitis. Segundo mecanismo: los altos niveles de mediadores inflamatorios, tales como el factor de necrosis tumoral alfa (TNF-a), IL-6, IL-8 e IL-18, pueden estar asociados con un riesgo elevado de disfunción endotelial, teniendo en cuenta que los niveles de los marcadores inflamatorios se encuentran también aumentados en sujetos con periodontitis. Tercer mecanismo: los patógenos periodontales o sus productos podrían afectar directamente la función endotelial. ${ }^{4}$

Se ha descubierto que los microorganismos periodontales, como Porphyromonas gingivalis o su toxina, podrían acceder a la circulación sanguínea, invadiendo de manera directa la pared arterial para después provocar inflamación vascular, ateroesclerosis y afectar directamente la función endotelial. ${ }^{7}$

La disfunción endotelial resultante de la inflamación crónica se asocia con un aumento en las especies reactivas de oxígeno.? El exceso de producción de las especies reactivas de oxígeno conduce a una disminución del óxido nítrico. La actividad deteriorada del óxido nítrico en los lechos vasculares y el daño al sistema antioxidante pueden contribuir a la disfunción endotelial en pacientes con periodontitis. El factor de necrosis tumoral $\alpha$ y otras citocinas producidas por el estrés inflamatorio podrían 
inhibir la actividad del óxido nítrico sintasa al impedir la degradación del inhibidor endógeno del óxido nítrico sintasa. El deterioro de la función eréctil en la PD podría derivarse del daño de la relajación del músculo liso dependiente del endotelio y la subsiguiente insuficiencia vascular. ${ }^{16}$

Se han realizado múltiples estudios en varias partes del mundo para explorar esta relación e informar sobre la posible asociación entre la PD y la DE en los últimos años. A continuación, se expone un cuadro con diez estudios realizados en la última década (Tabla 1).

\section{DISCUSIÓN}

La revisión del sistema y el metaanálisis realizado por Zhou y colaboradores, ${ }^{24}$ en 2016, evidencian que la PD aumenta la aparición de DE, y la PD podría tener implicaciones clínicas importantes para la estratificación del riesgo de disfunción eréctil. Sin embargo, recomiendan más estudios prospectivos con un mejor diseño para proporcionar evidencia más sólida sobre la relación entre ambas enfermedades.

De igual forma, Bizarro y Loos ${ }^{25}$ en 2019 realizaron una revisión y presentación de la evidencia disponible actual para determinar si existe un posible vínculo entre PD y DE. Demostraron que la condición del sistema vascular es peor en pacientes con periodontitis que en aquellos que no la tienen. Con base en esta evidencia, es posible deliberar que la periodontitis también puede estar asociada con la DE y, más específicamente, con la impotencia vasculogénica, una condición resultante de los cambios ateroscleróticos de las arterias y otros vasos sanguíneos. Diez estudios transversales y dos metaanálisis apuntaron claramente hacia este enlace. Además, un estudio de tratamiento a corto plazo sugiere que el tratamiento de la periodontitis puede disminuir las quejas de DE según la evaluación de los cuestionarios solos.

Tabla 1: Estudios sobre la relación entre periodontitis y disfunción eréctil en la última década.

\begin{tabular}{|c|c|c|c|c|c|c|c|}
\hline & Estudio & $\begin{array}{l}\text { País de } \\
\text { estudio }\end{array}$ & $\begin{array}{l}\text { Número de } \\
\text { sujetos }\end{array}$ & Rango de edad & $\begin{array}{c}\text { Método de diagnóstico } \\
\text { para } \mathrm{DE}\end{array}$ & $\begin{array}{c}\text { Métodos de } \\
\text { diagnóstico } \\
\text { para PD }\end{array}$ & $\begin{array}{c}\text { Resultados } \\
\text { primarios }\end{array}$ \\
\hline 1 & $\begin{array}{l}\text { Sharma et al. } \\
\qquad 2011^{17}\end{array}$ & India & $70(70 / 0)$ & $35 \pm 4$ & $\begin{array}{l}\text { Cuestionario/Ultrasonido } \\
\text { Doppler }\end{array}$ & $\begin{array}{c}\text { Examinación } \\
\text { dental/Rayos X }\end{array}$ & Positivo \\
\hline 2 & $\begin{array}{l}\text { Zuo et al. } \\
2011^{18}\end{array}$ & China & $\begin{array}{l}10 \text { ratas macho } \\
(5 / 5)\end{array}$ & 12 semanas & Análisis de Western Blot & $\begin{array}{l}\text { Inducción de } \\
\text { periodontitis }\end{array}$ & Positivo \\
\hline 3 & $\begin{array}{l}\text { Keller et al. } \\
2012^{19}\end{array}$ & Taiwán & $\begin{array}{c}195,336 \\
(32,856 / 162,480)\end{array}$ & $49 \pm 13$ & Cuestionario & $\begin{array}{l}\text { Examinación } \\
\text { dental/Rayos X }\end{array}$ & Positivo \\
\hline 4 & $\begin{array}{l}\text { Eltas et al. } \\
2013^{20}\end{array}$ & Turquía & $120(60 / 60)$ & $37 \pm 7$ & Cuestionario & $\begin{array}{c}\text { Examinación } \\
\text { dental }\end{array}$ & Positivo \\
\hline 5 & $\begin{array}{l}\text { Oguz et al. } \\
2013^{21}\end{array}$ & Turquía & $162(80 / 82)$ & $35 \pm 5$ & Cuestionario & $\begin{array}{c}\text { Examinación } \\
\text { dental }\end{array}$ & Positivo \\
\hline 6 & $\begin{array}{l}\text { Uppal et al. } \\
2014^{22}\end{array}$ & India & $53(53 / 0)$ & $25-40$ & $\begin{array}{c}\text { Diagnóstico basado en el hos- } \\
\text { pital (método no informado) }\end{array}$ & $\begin{array}{l}\text { Examinación } \\
\text { dental/Rayos X }\end{array}$ & Positivo \\
\hline 7 & $\begin{array}{l}\text { Matsumoto et } \\
\text { al. } 2014^{1}\end{array}$ & Japón & $88(88 / 0)$ & $51 \pm 17$ & Cuestionario & $\begin{array}{c}\text { Examinación } \\
\text { dental }\end{array}$ & Positivo \\
\hline 8 & $\begin{array}{l}\text { Tsao et al. } \\
2015^{13}\end{array}$ & Taiwán & $\begin{array}{c}15,315 \\
(5,105 / 10,210)\end{array}$ & $48 \pm 13$ & Cuestionario & $\begin{array}{c}\text { Examinación } \\
\text { dental }\end{array}$ & Positivo \\
\hline 9 & $\begin{array}{l}\text { Lee et al. } \\
2015^{23}\end{array}$ & Corea & $\begin{array}{c}513,258 \\
(2,732 / 510,526)\end{array}$ & Desconocido & Cuestionario & $\begin{array}{c}\text { Examinación } \\
\text { dental }\end{array}$ & Positivo \\
\hline 10 & $\begin{array}{c}\text { Martín et al. } \\
2018^{9}\end{array}$ & España & $158(80 / 78)$ & $53 \pm 9$ & Cuestionario & $\begin{array}{c}\text { Examinación } \\
\text { dental }\end{array}$ & Positivo \\
\hline
\end{tabular}

$\mathrm{DE}=$ disfunción eréctil, $\mathrm{PD}=$ periodontitis. 


\section{Tabla 2: Conclusiones de estudios sobre la relación entre periodontitis y disfunción eréctil en la última década.}

Estudio

1 Sharma et al. $2011^{17}$

2 Zuo et al. $2011^{18}$

$3 \quad$ Keller et al. $2012^{19}$

$4 \quad$ Eltas et al. $2013^{20}$

$5 \quad$ Oguz et al. $2013^{21}$

$6 \quad$ Uppal et al. $2014^{22}$

7 Matsumoto et al. $2014^{1}$

8 Tsao et al. $2015^{13}$

$9 \quad$ Lee et al. $2015^{23}$

10 Martín et al. $2018^{9}$
Se puede plantear la hipótesis de que existe una asociación entre la DE vasculogénica y la PD en varones jóvenes. Sin embargo, se justifica un estudio a gran escala con análisis de confusión y seguimiento longitudinal La función de la erección del pene se ve afectada por la PD. La disminución en la expresión de eNOS y la actividad NOS en el tejido cavernoso del pene causada por un estado inflamatorio sistémico leve en la PD puede ser uno de los factores de riesgo importantes de la DE

Nuestro estudio demostró una asociación entre la DE y el diagnóstico previo de PD

Los hallazgos del estudio sugieren que el tratamiento periodontal puede proporcionar beneficios adicionales en la mejora de la DE. Sin embargo, se necesitan más estudios para comprender los mecanismos de interacción entre estas enfermedades

Nuestros resultados han sugerido que la PD tenía una alta asociación con la DE en adultos jóvenes de 30 a 40 años. Creemos que será beneficioso considerar la enfermedad periodontal como una condición clínica causante de la DE en tales pacientes

Se puede concluir que la PD y la DE están asociadas entre sí. Sin embargo, se necesitan más estudios a gran escala con análisis de confusión y seguimiento longitudinal para explorar el vínculo entre estas dos enfermedades En conclusión, existe una correlación estadísticamente significativa entre PD y DE. El presente estudio sugiere que la DE está relacionada con el daño causado por la disfunción endotelial y los cambios inflamatorios sistemáticos asociados con la PD

Este estudio utilizó datos basados en la población de Taiwán para confirmar una nueva relación entre la DE y las enfermedades periodontales crónicas previas. También se descubrió que la extracción dental parece atenuar el desarrollo de la disfunción eréctil, excepto en la población más joven de mediana edad. La PD desempeña un papel clave en el proceso patológico de la DE y es un factor de riesgo que es independiente de otras morbilidades y factores de riesgo para la DE. Estos hallazgos informarán futuros estudios sobre la asociación entre inflamación crónica, disfunción endotelial, PD y DE. Además, este trabajo destaca la necesidad de clínicas que atiendan a pacientes con PD para evaluar la función eréctil

Se encontraron correlaciones significativas y positivas entre la PD y todas las comorbilidades relacionadas con el estilo de vida, excepto el infarto de miocardio, utilizando datos de la base de datos de cohortes del Seguro Nacional de Salud de Corea de 2002 a 2013. En particular, las enfermedades relacionadas con el estilo de vida, la DE y la osteoporosis están estrechamente relacionadas con la PD

Los pacientes con DE mostraron peor condición periodontal. La PD crónica parece desempeñar un papel clave como factor de riesgo en la patogénesis de la DE, independientemente de otras morbilidades

$\mathrm{DE}=$ disfunción eréctil, $\mathrm{PC}=$ precondición, $\mathrm{eNOS}=$ endothelial nitric oxide synthase, $\mathrm{PD}=$ periodontitis, $\mathrm{NOS}=$ nitric oxide synthase .

La expresión del óxido nítrico sintasa endotelial (eNOS) en el tejido del pene es una precondición (PC) que tiene un papel importante en el mecanismo de erección.

\section{RESULTADOS}

Diez estudios de casos y controles encontraron asociaciones positivas significativas entre estas dos condiciones (Tabla 2).

\section{CONCLUSIÓN}

La evidencia de la literatura y múltiples estudios indican un vínculo positivo entre la PD y la DE. Es con lo anterior que el médico y el cirujano dentista deben trabajar de la mano para tratar y controlar a los pacientes que presenten estas dos condiciones, no sólo por su posible relación entre ambas, sino también con otras enfermedades sistémicas más graves.

\section{BIBLIOGRAFÍA}

1. Matsumoto S, Matsuda M, Takekawa M, Okada M, Hashizume K, Wada $\mathrm{N}$ et al. Association of ED with chronic periodontal disease. Int J Impot Res. 2014; 26 (1): 13-15. 
2. Hajishengallis G. Periodontitis: from microbial immune subversion to systemic inflammation. Nat Rev Immunol. 2015; 15 (1): 30-44

3. Clavería-Clark RA, Rodríguez-Guerrero K, Peña-Sisto M. Enfermedad periodontal y disfunción eréctil: iexiste asociación directa? MediSan. 2015; 19 (11): 1399-1407.

4. Correa E, Kurt-Espinoza DA, Alonso-Espinoza D, Ariza T, Alarcon M. Enfermedad periodontal y disfunción eréctil. Rev Clin Periodoncia Rehabil Oral. 2015; 8 (1): 93-97.

5. Kalakondaa B, Koppolua P, Baroudia K, Mishra A. Periodontal systemic connections-novel associations-a review of the evidence with implications for medical practitioners. Int J Health Sci (Qassim). 2016; 10(2): 293-307.

6. Rodríguez-Fernández AA, Jiménez CA, Herrera FJ. Disfunción eréctil post-enfermedad periodontal. Rev Cient Univ Odontol Dominic. 2015; 2 (1): 39-50.

7. Singh VP, Nettemu SK, Nettem S, Hosadurga R, Nayak SU. Oral health and erectile dysfunction. J Hum Reprod Sci. 2017; 10 (3): 162-166.

8. Buitrago-Gómez N, Smith-Husbands J, García-Perdomo HA. Mecanismo de Frank Starling: una visión aplicada en la erección del pene. Urol Colomb. 2019; 28 (2): 183-188.

9. Martín A, Bravo M, Arrabal M, Magán-Fernández A, Mesa F. Chronic periodontitis is associated with erectile dysfunction. A case-contro study in European population. J Clin Periodontol. 2018; 45(7): 791-798.

10. Uribe-Arcila JF. Parámetros hemodinámicos en el proceso normal de la erección. Urol Colomb. 2015; 24 (2): 101-105.

11. Rodríguez-Rodríguez T. Disfunción sexual eréctil: zapenas una dificultad de los órganos sexuales? Finlay. 2017; 7 (3): 225-228.

12. Wang Q, Kang J, Cai $X$, Wu Y, Zhao L. The association between chronic periodontitis and vasculogenic erectile dysfunction: a systematic review and metaanalysis. J Clin Periodontol. 2016; 43 (3): 206-215.

13. Tsao CW, Liu CY, Cha TL, Wu ST, Chen SC, Hsu CY. Exploration of the association between chronic periodontal disease and erectile dysfunction from a population-based view point. Andrologia. 2014; 47 (5): 513-518.

14. Secretaría de Salud. Sabes qué es la disfunción eréctil [Internet], México: Gobierno de México [Fecha de publicación: 22 de marzo de 2017. Fecha de consulta: 24 de diciembre de 2019]. Disponible en: https://www.gob.mx/salud/articulos/disfuncion-erectil.
15. Maza-Bustamante V. Hasta la vista, disfunción eréctil [Internet], México: Milenio [Fecha de publicación: 17 de enero de 2015. Fecha de consulta: 24 de diciembre de 2019]. Disponible en: https://www.milenio.com/blogs/qrr/hasta-la-vista-disfuncion-erectil.

16. Liu LH, Li EM, Zhong SL, Li YQ, Yang ZY, Kang R et al. Chronic periodontitis and the risk of erectile dysfunction: a systematic review and meta-analysis. Int J Impot Res. 2017; 29 (1): 43-48.

17. Sharma A, Pradeep AR, Raju PA. Association between chronic periodontitis and vasculogenic erectile dysfunction. J Periodontol. 2011; 82 (12): 1665-1669.

18. Zuo Z, Jiang J, Jiang R, Chen F, Liu J, Yang $\mathrm{H}$ et al. Effect of periodontitis on erectile function and its possible mechanism. J Sex Med. 2011; 8 (9): 2598-2605.

19. Keller JJ, Chung SD, Lin HC. A nationwide population-based study on the association between chronic periodontitis and erectile dysfunction. J Clin Periodontol. 2012; 39 (6): 507-512.

20. Eltas A, Oguz F, Uslu MO, Akdemir E. The effect of periodontal treatment in improving erectile dysfunction: a randomized controlled trial. J Clin Periodontol. 2013; 40 (2): 148-154.

21. Oguz F, Eltas A, Beytur A, Akdemir E, Uslu MÖ, Güne, A. Is there a relationship between chronic periodontitis and erectile dysfunction? J Sex Med. 2013; 10 (3): 838-843.

22. Uppal RS, Bhandari R, Singh K. Association between erectile dysfunction and chronic periodontitis: a clinical study. Indian J Dent Res. 2014; 25 (4): 430-433.

23. Lee JH, Lee JS, Park JY, Choi JK, Kim DW, Kim YT et al. Association of lifestylerelated comorbidities with periodontitis: a nationwide cohort study in Korea. Medicine (Baltimore). 2015; 94 (37): 1-5.

24. Zhou X, Cao F, Lin Z. Updated evidence of association between periodontal disase and incident erectile dysfunction. J Sex Med. 2019; 16 (1): 61-69.

25. Bizzarro S, Loos BG. The link between periodontitis and erectile dysfunction: a review. Br Dent J. 2019; 227 (7): 599-603.

\section{Correspondencia: \\ Dra. Celia Elena del Perpetuo Socorro \\ Mendiburu-Zavala \\ E-mail: cel_mendi@hotmail.com}

Conflicto de intereses: Los autores declaran no tener ningún conflicto de intereses. 